\title{
Solitary Intraparotid Facial Nerve Plexiform Neurofibroma
}

\author{
Massimo Mesolella1, Antonella Miriam Di Lullo1, Filippo Ricciardiello1*, Flavia Oliva1, \\ Annalisa Pianese ${ }^{1}$, Gabriella Misso' ${ }^{2}$, Maurizio Iengo ${ }^{1}$ \\ ${ }^{1}$ Department of Head and Neck, Otolaryngology Unit, "Federico II" University, Naples, Italy \\ ${ }^{2}$ Department of Biochemistry, Biophysics and General Pathology, Second University of Naples, Naples, Italy \\ Email: filipporicciardiello@virgilio.it
}

Received 4 July 2014; revised 3 August 2014; accepted 1 September 2014

Copyright (C) 2014 by authors and Scientific Research Publishing Inc.

This work is licensed under the Creative Commons Attribution International License (CC BY). http://creativecommons.org/licenses/by/4.0/

(c) $\underset{\mathrm{EY}}{0}$ Open Access

\begin{abstract}
Solitary intraparotid facial nerve plexiform neurofibromas are extremely rare. These tumors arise from Schwann cells. The plexiform variant is recognized by tortuous and multinodular gross and microscopic lesions. It has a high risk of malignant transformation. We report a case of a solitary plexiform neurofibroma in a 5-year-old Italian male, who initially presented a right parotid mass of four-month duration. He had not pain, trismus, facial weakness or previous trauma. There was not familiar history of Von Recklinghausen's disease. TC and MRI scans revealed a mass in the superficial lobe of the parotid gland. A partial parotidectomy was performed. Histopatological examination indicated plexiform neurofibroma. The incidence, presentation, diagnosis and surgical treatment of this lesion are discussed. In our case, the tumor could be readily separated from the main trunk, and facial movement was completely preserved owing to the rich neural network around the mass.
\end{abstract}

\section{Keywords}

Intraparotid Facial Nerve, Plexiform Neurofibroma, Parapharyngeal Space

\section{Introduction}

Solitary intraparotid facial nerve plexiform neurofibromas are extremely rare. Plexiform neurofibromas (PNFs) are benign tumors originating from subcutaneous or visceral peripheral nerves and involving multiple fascicles. These tumors are either present at birth or develop within the first year of life.

Generally plexiform neurofibromas occur almost exclusively in patients with neurofibromatosis type 1 (NF1),

"Corresponding author.

How to cite this paper: Mesolella, M., Di Lullo, A.M., Ricciardiello, F., Oliva, F., Pianese, A., Misso, G. and lengo, M. (2014) Solitary Intraparotid Facial Nerve Plexiform Neurofibroma. International Journal of Clinical Medicine, 5, 1125-1129. 
an autosomal dominant disorder caused by genetic alterations of a tumor suppressor gene, which encodes the protein called neurofibromin-NF1 [1]. They can occur also as solitary neurofibromas, or as multiple neurofibromas without NF1. However the association between NF1 and plexiform neurofibroma is the most common: in fact the plexiform neurofibroma is considered as a patognomonic criterion for NF1 diagnosis. The solitary plexiform neurofibroma is not associated with NF1 [2] [3].

Plexiform neurofibromas are locally invasive, non metastating and generally categorized by location. They can arise in various parts of the body such as anterior mediastinal masses, sciatic nerve lesions with pelvic extension, or perirectal plexiform and uterine tumors, all leading to severe clinical sequels. Plexiform neurofibromas of the head, neck, and face are the most common, followed by lesion of the spine, extremities, mediastinum and abdomen [4]-[6]. Tumors occurring in the head and neck often lead to facial disfigurement, pain and functional deficits, and thus require surgical intervention. However, due to the large size and involvement of multiple fascicles of nerves as well as multiple tissue layers, satisfactory resection is often not possible without functional deficits resulting. Remains of the tumor may re-grow after the operation. The growth rates and patterns of plexiform neurofibromas vary largely and are not predictable [7]. Plexiform neurofibroma can originate malignant peripheral nerve sheath tumor, which occurs in $2 \%$ - $5 \%$ of patients with plexiform neurofibroma [8]. Malignat peripheral nerve sheath tumour is the main cause of death.

The peripheral portion of the facial nerve within the parotid gland gives five terminal branches (parotid plexus). The majority of the neurofibromas arising in this area, specifically the plexiform variant, have been described in children with NF1 [2] [8]. Some Authors believe that the solitary plexiform neurofibroma probably represents the segmental form of NF1 due to a later somatic mutation [9] [10].

In English literature it is reported that primitive neurogenic neoplasms occur more frequently in the last four cranial nerves and in sympathetic and parasympathetic chains located in the parapharyngeal spaces [11]. Intracranial and intra-parotid neoplastic involvement of facial nerve is much less common [8]. These neoplasms represent the $0.5 \%-0.6 \%$ of all human tumors [11].

Surgery remains the only viable therapy in patients with disability, intractable pain or disfigurement [6]. The preoperative planning and surgical management of plexiform neurofibromas involves two principal concerns: the timing of the operation and the extent of resection, considering also recurrence probability and potential loss of function [6].

We present this solitary plexiform neurofibroma case, the second intraparotid facial nerve pediatric, reported in English-language literature.

\section{Case Report}

A 5-year-old Italian male was referred for evaluation and management of a progressively enlarging right parotid mass of 4 months duration. He didn't have any pain, trismus, facial weakness or previous trauma. There wasn't stigmata or familiar history of von Recklinghausen's disease or Neurofibromatosis type 1 (NF1). On physical examination, a $(2 \mathrm{~cm} \times 2 \mathrm{~cm})$ firm, mobile mass was palpable slightly superior to the right angle of the mandible. On palpation, the swelling was plexiform, resembling a "bag of worms". There was no associated lymphadenopathy or facial nerve weakness.

A high frequency ultrasonography visualized an enlarging right parotid mass $(2 \mathrm{~cm} \times 1.7 \mathrm{~cm})$ in the superficial glandular lobe. In particular ultrasound appearance showed heterogeneity and poor definition of margins: in fact it visualized an irregular posterior heterogeneous margin and it was not possible to examine the deep one on ultrasound. In addition in the central part of the mass there was a tortuous hypoechoic area with irregular margins. The glandular duct was dilated. A CT scan with contrast localized the mass to the superficial lobe of the parotid gland. The mass had a central area of attenuation and was non-enhancing with the injection of contrast material. A MRI with contrast was better able to localise the mass of the parotid gland with variable morphology and central areas of low-signal density (Figure 1(a) and Figure 1(b)). Then a fine needle aspiration for cytology (FNA) was performed and was conclusive for the presence of "Schwann cells and fibroblast proliferation and myxoid stroma”, so a diagnosis of superficial plexiform neurofibroma was considered.

A partial parotidectomy was performed for excision under general anesthesia (Figure 2(a) and Figure 2(b)). Intraoperatively, the mass was found to be well-circumscribed and tan coloured (Figure 3). The main trunk of the facial nerve appeared to be adherent to the mass and was carefully dissected off. The histology revealed a plexiform neufibroma with a characteristic multinodular lesion: "macroscopic aspects: a $9 \times 4 \times 2 \mathrm{~cm}$ greywhite mass, with alternance of areas of elastic and myxoid consistency; microscopic aspects: oval/spindle- 


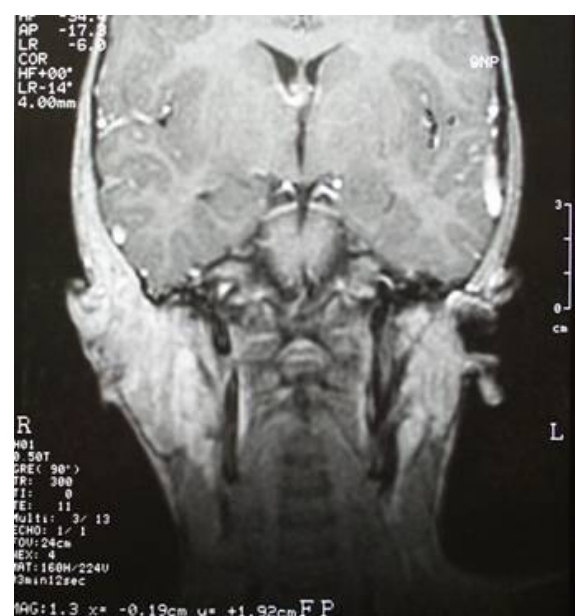

(a)

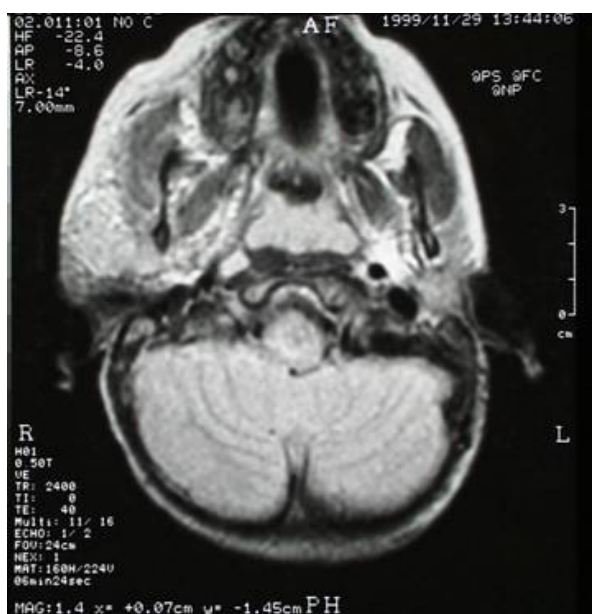

(b)

Figure 1. MRI with contrast was better able to localise the mass of the parotid gland (white arrows) with variable morphology and central areas of low-signal density. (a) Coronal T1-weighted MRI. (b) Axial T1-weighted MRI.

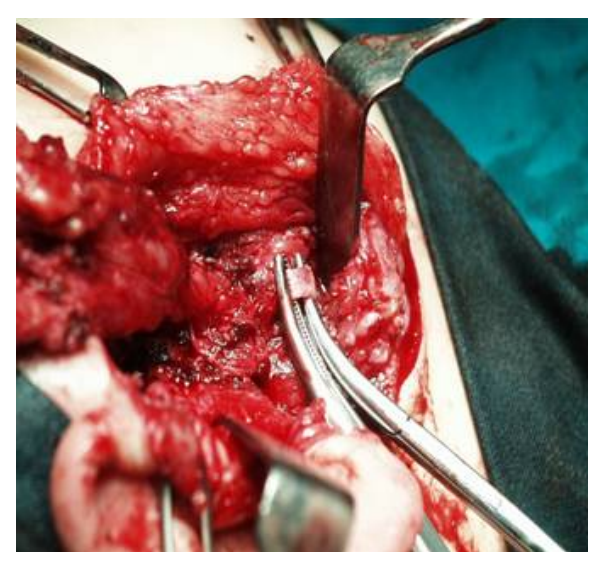

(a)

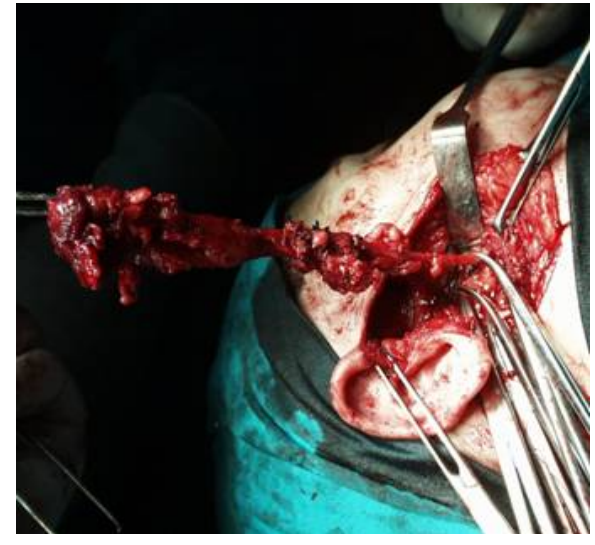

(b)

Figure 2. Intra-operative view. (a) A capsulated mass with its branches was identified. (b) The neoplasm was isolated following its capsule and resected.

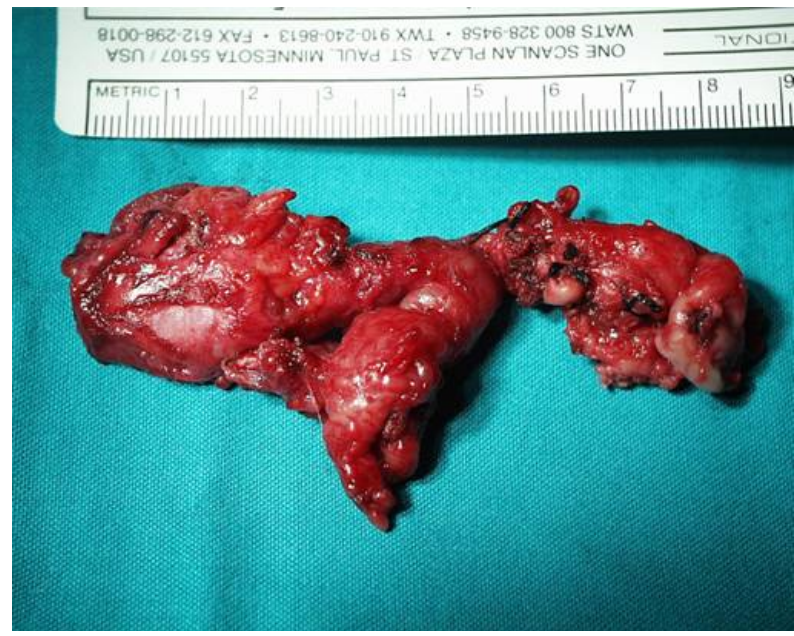

Figure 3. Surgical specimen (plexiform neufibroma resected). 
shaped Schwann cells, fibroblast proliferation and abundant myxoid stroma”.

No regrowth was detected after 1 year's follow-up and 2 year's one. No local recurrence was found 5 years after the operation.

\section{Discussion}

Plexiform neurofibroma is commonly diagnosed in children and rarely after adolescence. It can involve superficial tissue or deeper tissue and the most common site is the trunk (43\%), head and neck area (42\%) and limbs (15\%) [12]. Plexiform neurofibromas in the head and neck region can cause significant morbidity by interference with the function of a sensory organ (eye or ear), obstructive airway symptoms, swallowing difficulty, and speech problems. In addition, large head and neck plexiform neurofibromas cause disfigurement, which frequently leads to social withdrawal [6]. In our case, patient had only a right parotid mass without other symptoms as pain, trismus or facial weakness.

The tumor is described as gradually progressive, locally invasive and often disfiguring. Loss of function is possible due to neurovascular involvement with nerve compression [13]. In general, plexiform neurofibromas cause diffuse nodular enlargement of a nerve and its branches by a proliferation of Schwann cells in the nerve sheath across the length of a nerve [10]. There is also a $4 \%$ incidence of malignant transformation of plexiform neurofibroma.

Plexiform neurofibromas associated with NF1 are more frequent and tend to be larger, multiple and involve deep nerves [14]. The PNFs associated with NF1 have the potential to undergo sarcomatous change towards neurofibrosarcoma, or malignant schwannoma, which are the main cause of death in this group of patients [15] [16].

In literature, we found some cases of solitary plexiform neurofibroma, not associated with NF1 [2] [3] [10] [17] [18]. Rarely the solitary type of plexiform neurofibroma undergoes sarcomatous change and for this reason follow-up with the late development of features of NF1 is important [15].

Solitary lesions should be treated with excision because of recurrences are rare in these cases [10].

Neurogenic neoplasms of the facial nerve are uncommon and those involving the intraparotid portion of the facial nerve are considered even less common. Our case represents a unusual case on this kind of tumor for the infrequent location. In fact, the first pediatric case report of intraparotid facial nerve solitary plexiform neurofibroma was published by Souaid et al. in 2003 [2]. Other cases examine adult patients [3] or patients with NF1.

Surgery is the only effective option currently available for the treatment of plexiform neurofibroma. These tumors are in fact non-radiosensitive and given their slow growth rates; in addition, only limited benefits have been observed with chemotherapy. However, success of surgical intervention is limited by the infiltrating nature of the tumors, resulting in a high rate of tumor re-growth. Age, extent of resection and location of tumor are independent variables affecting the outcome of surgery for plexiform neurofibromas [6]. Extent of resection has proven prognostic significance, with greater extent of surgical resection predicting both lower risk of tumor progression and longer interval to progression. Total or subtotal excision of the tumor ensures recurrence rates less than $20 \%$ and $40 \%$ respectively while removal of less than $90 \%$ of the tumor mass leads to a recurrence rate of $60 \%$ or greater [19]. Permanent neurological deficits occur in approximately $5 \%$ of patients who undergo surgery for neurofibromas. In the head and neck region the incidence of neurological deficits approaches $10 \%$.

Many studies have shown that patients younger than 10 years and those with lesions affecting the head and neck are at greater risk for recurrence. So many surgeons operate infrequently plexiform neurofibromas of the head and neck region, particularly in pediatric patients. Although facial nerve preservation during surgery is unlikely and significant morbidity can result from their excision, when the separation appears difficult nerve grafting is recommended for larger defects and end-to-end anastomosis is recommended for small defects of the facial nerve [20].

We chose surgical treatment and we performed a partial parotidectomy under general anesthesia; the tumor could be readily separated from the main trunk and totally removed.

Facial movement was completely preserved owing to the rich neural network around the mass. There was not local recurrence after 5-year follow-up and there were not development of other possible NF1 features.

\section{Conclusions}

Solitary intraparotid facial nerve plexiform neurofibroma is a rare tumor. We present this case for its rare site, in 
a young patient without clinical features of neurofibromatosis.

Based on our experience, we believe that it is possible to operate with a total or sub-total resection a plexiform neurofibroma of head and neck region in children with minimal morbidity. This requires comprehensive preoperative planning, meticulous surgical technique and vigilant post-operative intensive care.

\section{References}

[1] Huson, S.M. (1989) Recent Developments in the Diagnosis and Management of Neurofibromatosis. Archives of Disease in Childhood, 64, 745-749. http://dx.doi.org/10.1136/adc.64.5.745

[2] Souaid, J.P., Nguyen, V.H., Zeitouni, A.G. and Manoukian, J. (2003) Intraparotid Facial Nerve Solitary Plexiform Neurofibroma: A First Paediatric Case Report. International Journal of Pediatric Otorhinolaryngology, 67, 1113-1115. http://dx.doi.org/10.1016/S0165-5876(03)00184-8

[3] Malcolm, E.K. and Lopes, M.B. (2002) April 2002: 35-Year-Old Healthy Man with Enlarging Right Parotid Mass. Brain Pathology, 12, 515-521. http://dx.doi.org/10.1111/j.1750-3639.2002.tb00470.x

[4] Enzinger, F.M. and Weiss, S.W. (1988) Soft Tissue Tumors. 2nd Edition, CV Mosby Co., St. Louis, 745-756.

[5] Wise, J.B., Patel, S.G. and Shah, J.P. (2002) Management Issues in Massive Pediatric Facial Plexiform Neurofibroma with Neurofibromatosis Type 1. Head \& Neck, 24, 207-211. http://dx.doi.org/10.1002/hed.10001

[6] Ransom, E.R., Yoon, C. and Manolidis, S. (2006) Single Stage near Total Resection of Massive Pediatric Head and Neck Plexiform Neurofibromas. International Journal of Pediatric Otorhinolaryngology, 70, 1055-1061. http://dx.doi.org/10.1016/j.ijporl.2005.10.025

[7] Friedrich, R.E., Schmelzle, R., Hartmann, M. and Mautner, V.F. (2005) Subtotal and Total Resection of Superficial Plexiform Neurofibromas of Face and Neck: Four Case Reports. Journal of Cranio-Maxillofacial Surgery, 33, 55-60. http://dx.doi.org/10.1016/j.jcms.2004.08.004

[8] Fadda, M.T., Verdino, G., Mustazza, M.C., Bartoli, D. and Iannetti, G. (2008) Intra-Parotid Facial Nerve Multiple Plexiform Neurofibroma in Patient with NF1. International Journal of Pediatric Otorhinolaryngology, 72, 553-557. http://dx.doi.org/10.1016/j.ijporl.2008.01.022

[9] Ruggieri, M. and Huson, S.M. (2001) The Clinical and Diagnostic Implications of Mosaicism in the Neurofibromatoses. Neurology, 56, 1433-1443. http://dx.doi.org/10.1212/WNL.56.11.1433

[10] Zwane, N.P., Noffke, C.E.E. and Raubenheimer, E.J. (2011) Solitary Oral Plexiform Neurofibroma: Review of Literature and Report of a Case. Oral Oncology, 47, 449-451. http://dx.doi.org/10.1016/j.oraloncology.2011.04.005

[11] de Campora, E., Radici, M. and de Campora, L. (1999) Neurogenic Tumors of the Head and Neck in Children. International Journal of Pediatric Otorhinolaryngology, 49, S231-S233. http://dx.doi.org/10.1016/S0165-5876(99)00166-4

[12] Waggoner, D.J., Towbin, J., Gottesman, G. and Gutmann, D.H. (2000) Clinic-Based Study of Plexiform Neurofibromas in Neurofibromatosis 1. American Journal of Medical Genetics, 92, 132-135. http://dx.doi.org/10.1002/(SICI)1096-8628(20000515)92:2<132::AID-AJMG10>3.0.CO;2-6

[13] Sehgal, V.N., Sharma, S. and Oberai, R. (2009) Evaluation of Plexiformneurofibroma in Neurofibromatosis Type 1 in 18 Family Members of 3 Generations: Ultrasonography and Magnetic Resonance Imaging a Diagnostic Supplement. International Journal of Dermatology, 48, 275-279. http://dx.doi.org/10.1111/j.1365-4632.2009.03999.x

[14] Friedman, J.M. (2002) Neurofibromatosis Type 1: Clinical Manifestations and Diagnostic Criteria. Journal of Child Neurology, 17, 548-554. http://dx.doi.org/10.1177/088307380201700802

[15] Neville, B.W., Damm, D.D., Allen, C.M. and Bouquot, J.E. (2002) Oral and Maxillofacial Pathology. 2nd Edition, WB Saunders Company, Philadelphia.

[16] Geller, M., Darrigo Jr., L.G., Filbo, A.B. and Ribeiro, M.G. (2009) Plexiform Neurofibroma in the Ear Canal of a Patient with Type I Neurofibromatosis. Brazilian Journal of Otorhinolaryngology, 75, 158.

[17] Fierek, O., Laskawi, R. and Kunze, E. (2006) Solitary Intraparotid Neurofibroma of the Facial Nerve. Symptomatology, Biology and Management. HNO, 54, 772-777. http://dx.doi.org/10.1007/s00106-005-1366-3

[18] Sharma, A., Sengupta, P. and Anjan, K.R. (2013) Isolated Plexiform Neurofibroma of the Tongue. Journal of Laboratory Physicians, 5, 127-129. http://dx.doi.org/10.4103/0974-2727.119867

[19] Needle, M.N., Cnaan, A., Dattilo, J., Chatten, J., Phillips, P.C., Shochat, S., et al. (1997) Prognostic Signs in the Surgical Management of Plexiform Neurofibroma: The Children's Hospital of Philadelphia Experience, 1974-1994. The Journal of Pediatrics, 131, 678-682. http://dx.doi.org/10.1016/S0022-3476(97)70092-1

[20] Hehar, S.S., Dugar, J. and Sharp, J. (1999) The Changing Faces of a Parotid Mass. The Journal of Laryngology \& Otology, 113, 938-941. http://dx.doi.org/10.1017/S0022215100145657 
Scientific Research Publishing (SCIRP) is one of the largest Open Access journal publishers. It is currently publishing more than 200 open access, online, peer-reviewed journals covering a wide range of academic disciplines. SCIRP serves the worldwide academic communities and contributes to the progress and application of science with its publication.

Other selected journals from SCIRP are listed as below. Submit your manuscript to us via either submit@scirp.org or Online Submission Portal.
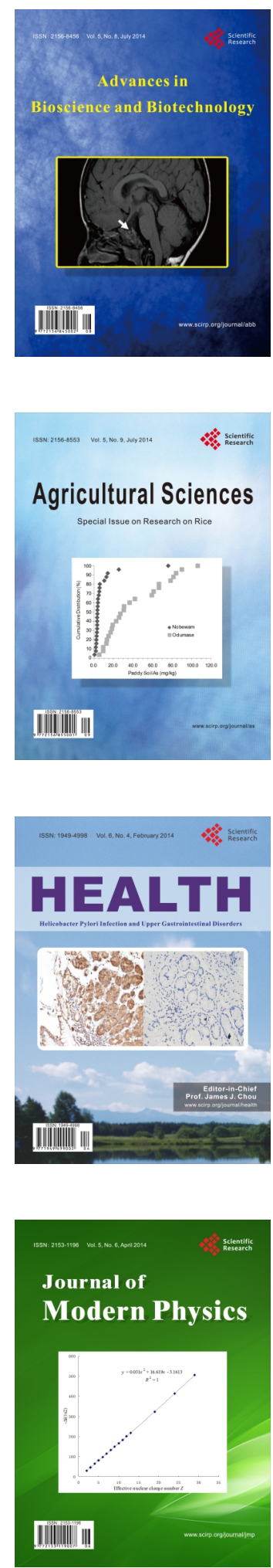
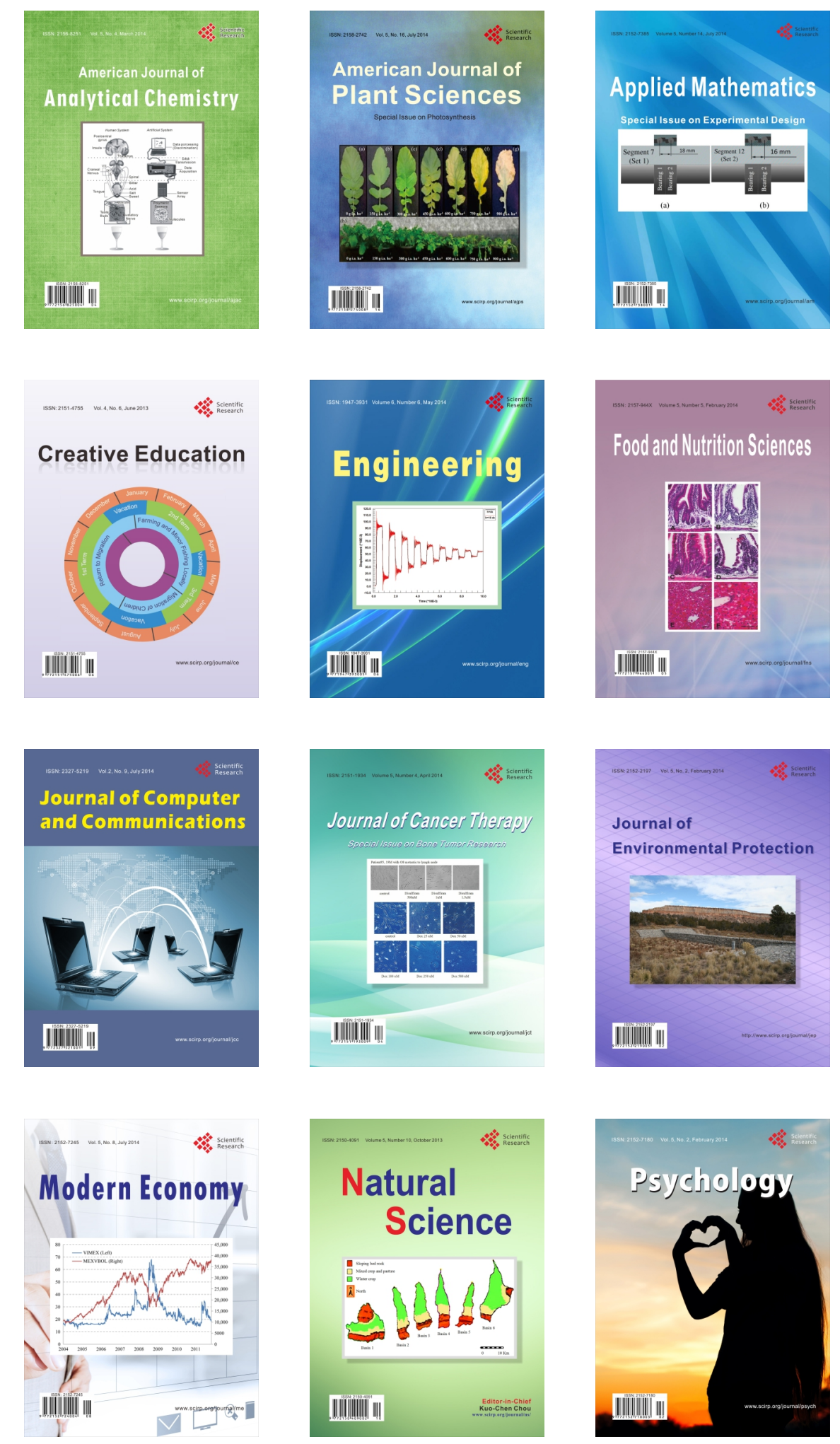